\title{
Влияние морфологии поверхности микрополосковой линии СВЧ на ее передаточные характеристики
}

\author{
() Н.А. Торхов ${ }^{1,2,3,4}$, А.А. Коколов ${ }^{2,5}$, Л.И. Бабак ${ }^{2}$ \\ ${ }^{1}$ Научно-исследовательский институт полупроводниковых приборов, \\ 634034 Томск, Россия \\ ${ }^{2}$ Томский университет систем управления и радиоэлектроники, \\ 634050 Томск, Россия \\ ${ }^{3}$ Томский государственный университет, \\ 634050 Томск, Россия \\ ${ }^{4}$ Севастопольский государственный университет, \\ 299053 Севастополь, Россия \\ ${ }^{5}$ Институт оптики атмосферы им. В.Е. Зуева Сибирского отделения Российской академии наук, \\ 634021 Томск, Россия \\ E-mail: trkf@mail.ru
}

Поступила в Редакцию 26 апреля 2020 г.

В окончательной редакции 18 мая 2020 г.

Принята к публикации 28 мая 2020 г.

\begin{abstract}
Определены основные морфологические параметры 50-омных $\mathrm{Au} / \mathrm{i}-\mathrm{GaAs}\{100\}$ тонкопленочных микрополосковых золотых СВЧ копланарных линий передач длиной $l_{W}$, влияющие на активное сопротивление их скин-слоя $R$ и индуктивность $L$. Получено, что латеральный характер распределения зерен и развитый рельеф их поверхностей приводит к возникновению дополнительных процессов рассеяния электронов как на границах зерен, так и на неоднородностях рельефа. Малый размер зерен, $d_{x}<133$ нм, на частотах $f>10$ ГГц переводит аномальный скин-эффект в нормальный. При этом нелинейная зависимость $R$ от $l_{W}$ в локальном приближении обеспечивается фрактальной геометрией рельефа поверхности и приповерхностной области копланарных линий передач, а нелинейная зависимость индуктивности $L$ от $l_{W}-$ не только фрактальными особенностями рельефа двухмерной поверхности копланарных линий передач, но и фрактальными особенностями трехмерного распределения ее зерен.
\end{abstract}

Ключевые слова: СВЧ линии, скин-эффект, морфология поверхности, фракталы, размерность ХаусдорфаБезиковича, $S$-параметры рассеяния, компактная модель, экстракция параметров.

DOI: $10.21883 /$ FTP.2020.11.50095.9416

\section{1. Введение}

В аномальном скин-эффекте условия $\delta \ll l_{\text {ball }}$ и $\delta \ll v_{\mathrm{F}} / \omega$ приводят к возникновению в скин-слое толщиной $\delta$ пространственной и частотной дисперсий сверхвысокочастотных (СВЧ) электромагнитных колебаний (СВЧ сигнал) и нарушению закона Ома $(\mathbf{j} \neq \sigma \mathbf{E})$. Здесь $l_{\text {ball }}=v_{\mathrm{F}} \tau_{\text {ball }}-$ среднее значение длины свободного, т.е. без рассеяния (баллистического), пробега низкоэнергетичных, с энергией $E_{0}<1$ эВ, электронов (при нормальных условиях $l_{\text {ball }} \sim 1000 \mathrm{HM}$, рис. $\left.\left.1, a\right)[1]\right)$, определяемой временем их баллистического переноса в однородном металлическом образце $\tau_{\text {ball }}(\omega=2 \pi f-$ циклическая частота, $v_{\mathrm{F}}$ - „фермиевская“ скорость). Увеличение частоты $f$ СВЧ сигнала при нормальных и пониженных температурах приводит к уменьшению глубины проникновения СВЧ поля под поверхность проводника $\delta \propto \omega^{(-1 / 3)}-$ поперечных размеров скин-слоя, увеличению его активного сопротивления $R$ и уменьшению индуктивности $L$ линии передачи [2]. При этом функциональные зависимости $R=R(f)$ и $L=L(f)$ в аномальном скин-эффекте во многом определяются состоянием металлической поверхности, притом что значение поверхностного импеданса $\xi(\operatorname{Re} \xi=R)$ ее скин-слоя практически не зависит от характера рассеяния электронов на кристаллической решетке [3]. Напомним, что для нормального скин-эффекта $\delta \propto \omega^{(-1 / 2)}$.

Исследования размерных (перколяционных) эффектов на постоянных и переменных электрических токах в металлических тонкопленочных системах были связаны в основном с изучением влияния их поперечных (перпендикулярно поверхности, т. е. плоскости $(x, y))$ размеров на особенности токопрохождения [4]. Между тем используемые в производстве электронных приборов реальные металлические пленки имеют значительные латеральные, в плоскости $(x, y)$, неоднородности, так как образованы мозаичной структурой кристаллитов нанозерен размерами 20-500 нм, разделенных между собой плохо проводящими промежутками - модель Маядаса-Шатцкеса [5,6]. В этом случае размеры латеральных неоднородностей металлических пленок могут достигать сотен микрометров [7,8], а нормальных, определяемых высотой неровностей рельефа, неоднородностей - сотен нанометров, которые для частот $>10$ ГГц уже соизмеримы с толщиной скин-слоя (например, для золота $\mathrm{Au} \delta(f=20$ ГГц $) \approx 550 \mathrm{нм}$, рис. $1, b[9])$ и влияние которых на аномальный скин- эффект в настоящее время практически не изучено. 

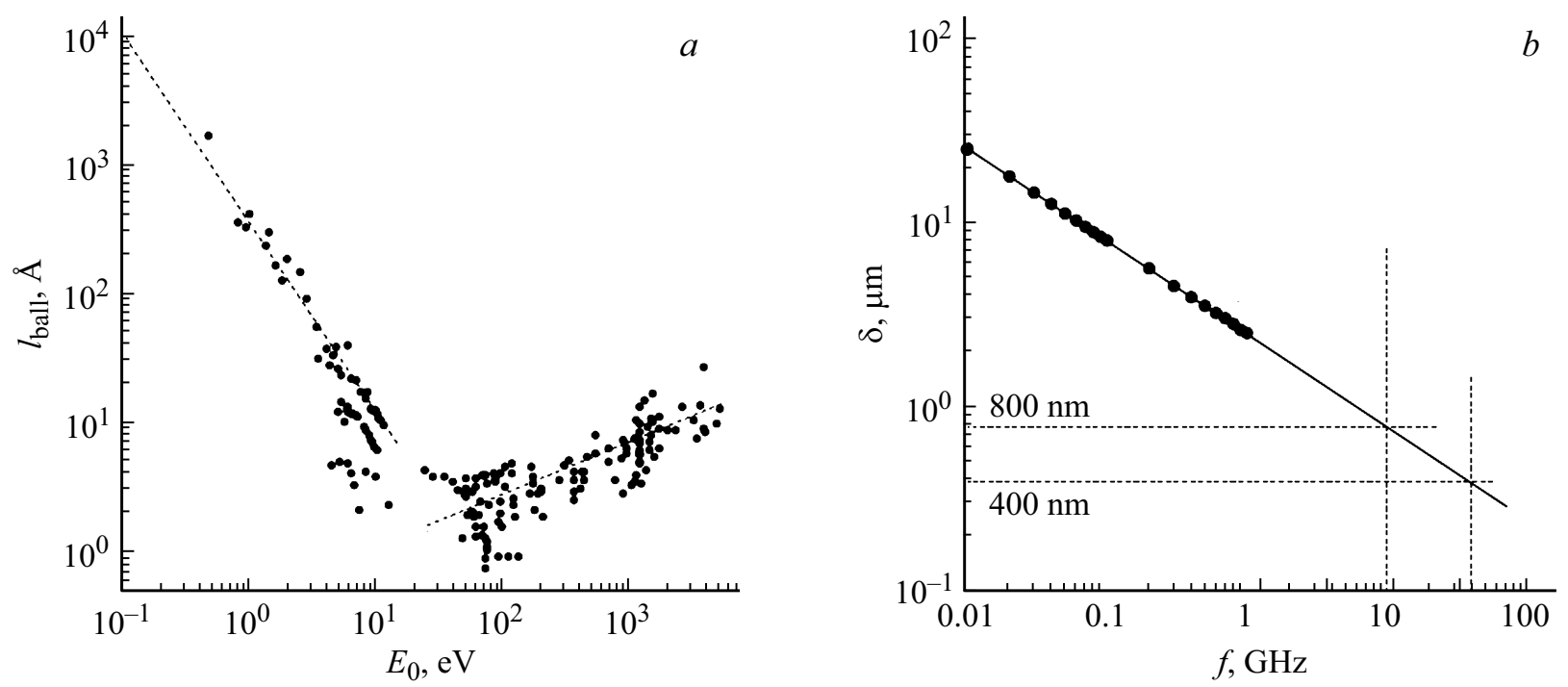

Рис. 1. Зависимость от энергии $E_{0}$ длины свободного пробега $l_{\text {ball }}$ электронов для $3 d-(\mathrm{Fe}-\mathrm{Cu})$ и $5 d-(\mathrm{Pt}, \mathrm{Au})$ металлов по данным [1] (a) и частотная зависимость толщины скин-слоя $\delta$ в однородном золотом образце $(b)$.

Из физических соображений следует, что такие латеральные неоднородности могут оказывать значительное влияние на движение электронов в скин-слое металлической пленки из-за их рассеяния на границах зерен, а также и на неровностях поверхности, что должно неизбежно отразиться на параметрах аномального скин-эффекта и, как результат, на качестве прохождения СВЧ сигнала.

Пространственное распределение таких неоднородностей в реальных металлических системах хорошо описывается математическим аппаратом фрактальной геометрии. Известно $[8,10]$, что пределы локальных приближений L - линейные размеры, до которых тонкопленочные металлические системы проявляют свойства статистически фрактальных объектов, - могут достигать сотен микрометров, что соизмеримо с длинами микрополосковых линий передач в интегральных схемах.

Таким образом, возникает значительный научнопрактический интерес в проведении экспериментальных исследований влияния геометрии поверхностей металлических пленок на характер распространения в них СВЧ сигналов.

\section{2. Объект исследований и методика эксперимента}

Исследование влияния геометрии Аu-поверхности микрополосковых копланарных линий передач (КЛП) СВЧ сигнала на их приборные характеристики в частотном диапазоне $0.1-50$ ГГц осуществлялось с использованием понятий меры М, метрических $\mathfrak{R}(\mathrm{X}, \rho)$ (состоящих из некоторого множества $\mathrm{X}=\left\{x_{i}\right\}$, в котором определено расстояние $\rho$ между любой парой элементов с аксиомами треугольника, симметрии и нулевого расстояния) и нормированных линейных функциональных пространств $\mathrm{S}(\Re, \mathrm{M})$ (далее - функциональных пространств), позволяющих с единых позиций рассматривать скалярные и векторные величины, непрерывные функции и числовые последовательности $[11,12]$.

Объектами исследований являлись 50-омные микрополосковые КЛП шириной $W=50$ мкм, длиной $l_{W}=100$, 200, 400, 800, 1600 и 3200 мкм, выполненные гальваническим золотом $\mathrm{Au}$ толщиной $d=3$ мкм с тонким адгезионным подслоем ванадия на полуизолирующей подложке арсенида галлия $\mathrm{GaAs}\{100\}$ толщиной $h=350$ мкм (рис. 2,a). Расстояние между „сигнальной“ и ,земляными“ копланарными линиями составляло $S_{W}=36$ мкм.

Ввиду того что КЛП образована совокупностью различных конструктивных элементов (,земляные“ и „сигнальные“ металлические линии, полуизолирующая полупроводниковая подложка), ее СВЧ характеристики удобно изучать с использованием компактной модели, представленной в виде эквивалентной схемы (ЭС) секции КЛП, состоящей из совокупности эквивалентных элементов с соответствующими электрическими связями между ними (рис. 2, $b$ ). Пассивные элементы ЭС $R$ и $L$ описывают последовательное сопротивление и индуктивность металлизации КЛП, а элементы $C$ и $G$ - емкость и проводимость подложки.

Измерения геометрии рельефа $h(x, y)$ и фазового контраста $\theta(x, y)$ поверхности металлических пленок осуществлялись на высоковакуумном атомно-силовом микроскопе (ACM) „Solver-HV“ производства NT-MDT (Москва, г. Зеленоград) с использованием кантилевера марки NSG10 (рис. 3). Радиус закругления острия иглы, покрытого карбидом вольфрама $\mathrm{W}_{2} \mathrm{C}$, составлял $r \approx 35$ нм. АСМ-измерения проводились в полуконтактном режиме сканирования с использованием двухпроходной методики при разрешении 256 пикселей по кадровой и строчной разверткам. 

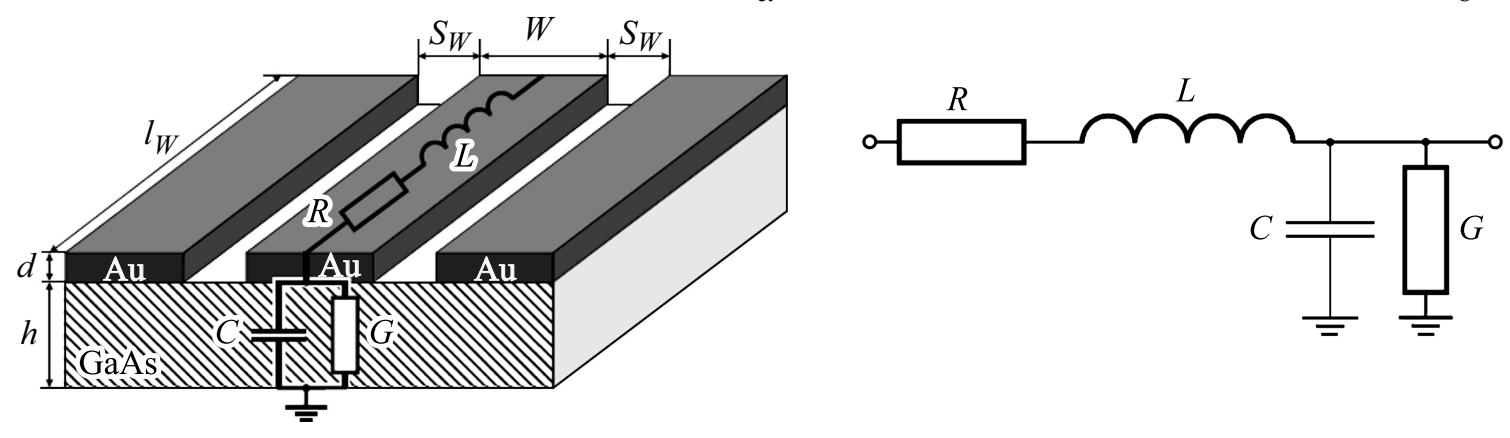

Рис. 2. Конструкция секции КЛП (a) и ее малосигнальная эквивалентная схема $(b)$.
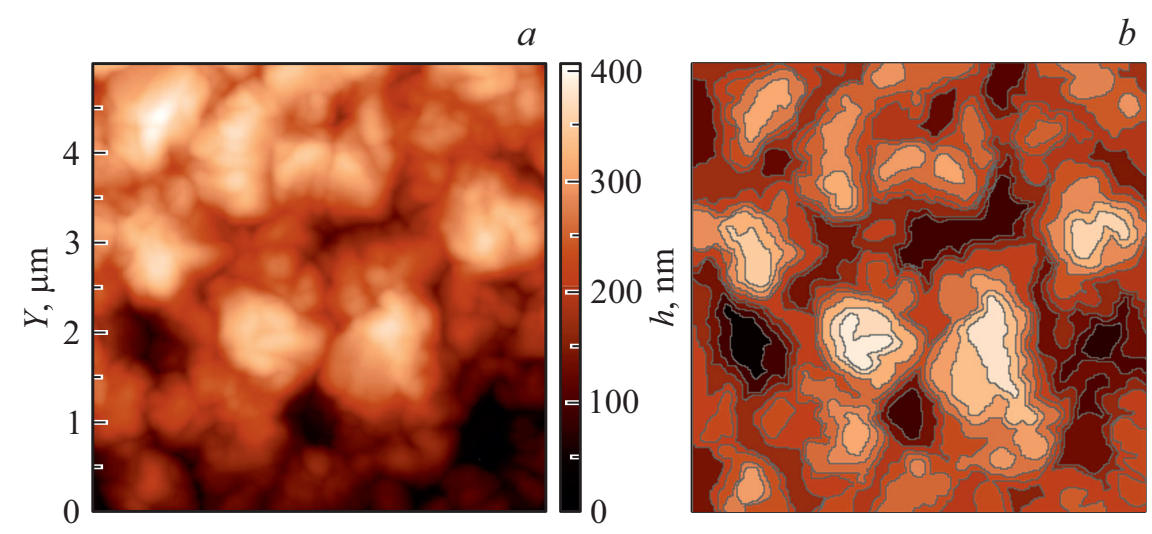

$b$

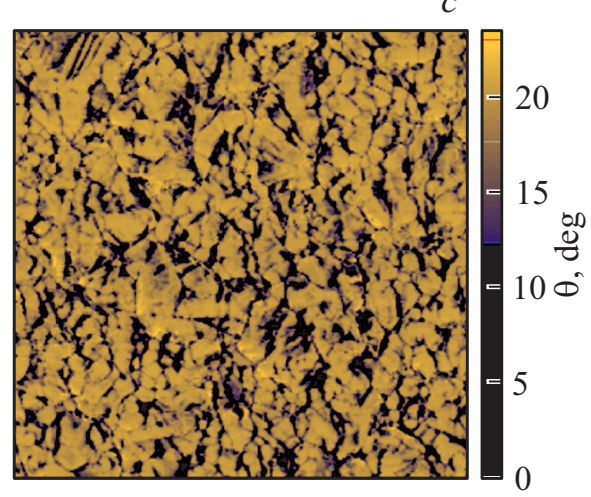

Рис. 3. АСМ-изображение рельефа поверхности $(a)$, его контурное изображение $(b)$ и распределение фазового контраста $(c)$.

Измерения малосигнальных $S$-параметров КЛП в частотном диапазоне 0.1-50ГГц выполнялись с использованием векторного анализатора цепей PNA-X N5245A производства Keysight Technologies на полуавтоматической зондовой станции Summit 12000 производства Cascade Microtech. Калибровка измерительных трактов портов осуществлялась по $S$-параметрам согласованием на проход („Thru“), на нагрузку 50 Ом (Load), на холостой ход („Ореп“) и на короткое замыкание („Short"). После этого проводилась процедура дэ-эмбеддинга (de-embedding) и экстракция параметров эквивалентных элементов ЭС секции КЛП $[13,14]$.

Хаусдорфова размерность $D_{\mathrm{H}}$ метрического пространства $\mathfrak{R}$ рельефа поверхности КЛП определялась методом подсчета кубов. Для самоподобных объектов в локальном приближении $\left(l_{W}<L\right)$ хаусдорфова размерность равна фрактальной размерности $D_{f}(1)$, а в глобальном $\left(l_{W} \geq L\right)$ приближении - топологической размерности объекта $D_{T}[15]$ :

$$
D_{f}=D_{T}+D_{S}=D_{T}+\ln (\eta) / \ln (\xi),
$$

где $D_{S}$ - размерность подобия, определяемая путем подсчета относительного увеличения количества островков $M$ (мер) в $\eta=M_{0} / M_{k}$ раз при относительном уменьшении линейных размеров измерительного масштаба (длины $l_{W}$ ) в $\xi=l_{W, 0} / l_{W, k}$ раз [15]. Топологическая размерность КЛП равнялась единице, $D_{T}=1$.

Согласно $[13,15]$, значения $D_{f}$, определенные на $\mathfrak{R}$ размерностей функциональных пространств активных сопротивлений $\mathrm{S}(M \equiv R)$ и индуктивностей $\mathrm{S}(M \equiv L)$, находились аналогичным методом (1), но только в качестве мер $M$ выбирались значения функционалов активных сопротивлений $M \equiv R$ и индуктивностей $M \equiv L$ копланарных линий.

\section{3. Результаты экспериментов}

Согласно рис. 3, $a$ размер неровностей рельефа $h$ исследуемого участка $5 \times 5$ мкм поверхности КЛП может заметно превышать $h>400 \mathrm{Hм}$, что уже соизмеримо с толщиной скин-слоя $(\delta(f=20$ ГГц $) \approx 550$ нм $)$. Из общих физических соображений понятно, что рассеяние электронов на таких неровностях поверхности должно оказывать заметное влияние на распространение СВЧ сигнала.

О характере распределения неровностей рельефа можно судить по контурному изображению исследуемого участка поверхности, полученному трассировкой неровностей рельефа (рис. $3, b$ ). Анализ контурных изображений показал, что они образованы вложенными друг в 

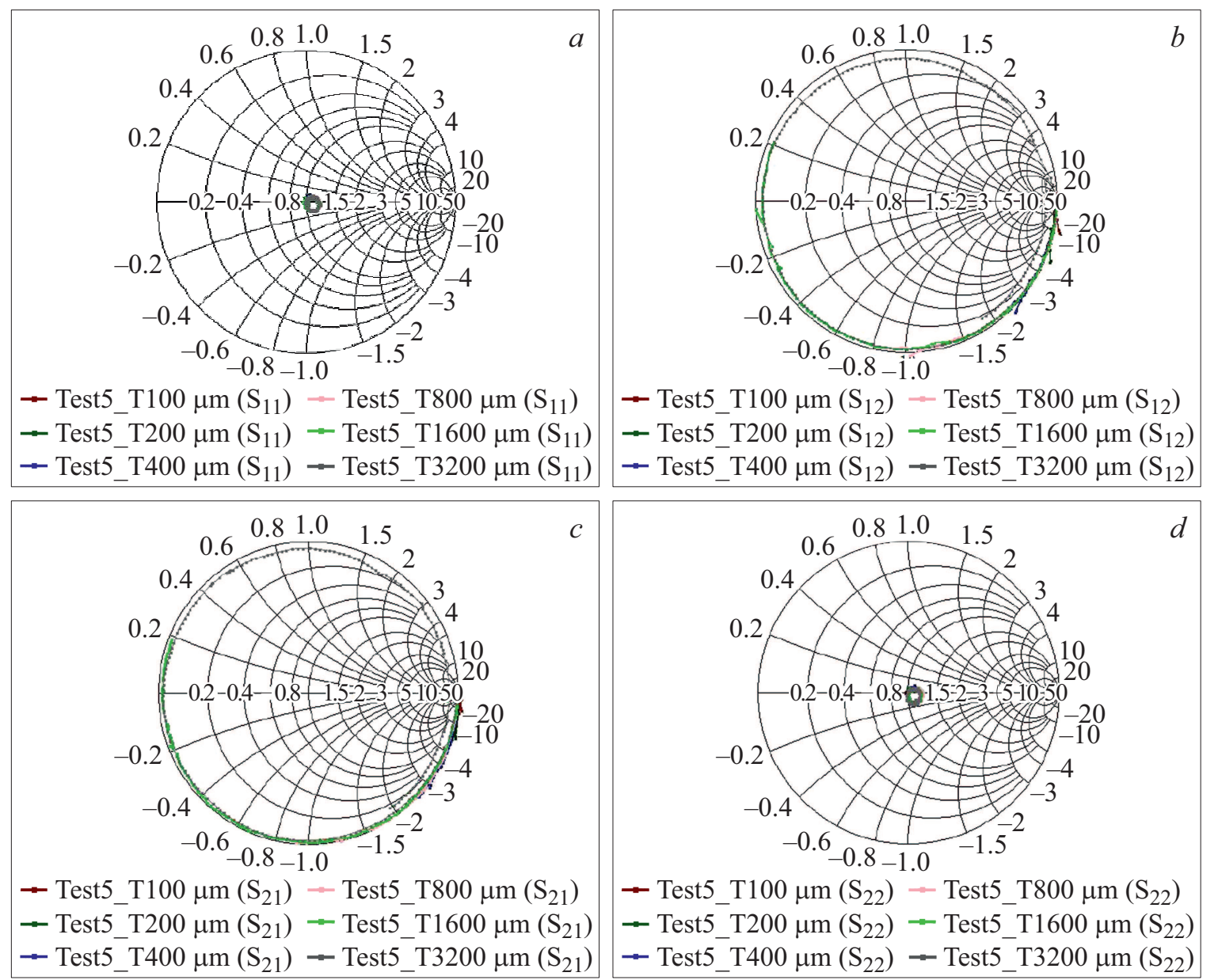

Рис. 4. Малосигнальные параметры рассеяния КЛП длиной $l_{W}=100,200,400,800,1600$ и 3200 мкм в частотном диапазоне $0.1-50$ ГГц: $\mathrm{S}_{11}(a), \mathrm{S}_{12}(b), \mathrm{S}_{21}-(c), \mathrm{S}_{22}-(d)$.

друга меньшими по размеру статистически самоподобными элементами предыдущих уровней подобия.

По АСМ-изображениям фазовых контрастов $\theta(x, y)$ можно судить о характере латерального расположения зерен (геометрии зеренной субструктуры). Согласно статистическому анализу, золотая пленка КЛП образована четырьмя множествами зерен, средние размеры $d_{x}$ которых приблизительно составляют 53, 72, 108, 133 нм и которые значительно меньше не только толщины скин-слоя,

$$
d_{x} \ll \delta(f=20 \text { ГГц }),
$$

но и среднего значения длины баллистического пробега электронов в однородном образце:

$$
d_{x} \ll l_{\text {ball }} \text {. }
$$

Неравенство (3) означает, что длина свободного пробега электронов $l_{\text {ball }}$ в исследуемых образцах ограничена размерами кристаллитов (зерен) и можно записать для исследуемых образцов $l_{\text {ball }} \approx d_{x}$. Таким образом, получается, что в используемом частотном диапазоне $l_{\text {ball }} \ll \delta$ и аномальный скин-эффект в исследуемых КЛП переходит в нормальный. Понятно, что геометрия распределения зерен в золотой пленке КЛП также будет влиять на характер рассеяния электронов и, как следствие, на качество распространения СВЧ сигнала.

Фрактальные размерности метрических пространств $\mathfrak{R}$ рельефа поверхности и зеренной субструктуры, определенные методом подсчета кубов, составили $D_{f s}=(2.11 \pm 0.08) \quad$ и $D_{f g}=(2.46 \pm 0.07)$ соответственно.

Таким образом, распределения центров рассеяния в виде неровностей рельефа и границ зерен описываются разными законами фрактальной геометрии, которые, как ожидается, будут определять интегральные характеристики электронного тока в скин- слое и в конечном итоге качество распространения СВЧ сигнала в КЛП.

Рассмотрим, как все-таки прохождение СВЧ-сигнала связано с фрактальной субструктурой КЛП.

Частотные зависимости малосигнальных $S$-параметров рассеяния, исследуемых КЛП длиной $l_{W}=100$, $200,400,800,1600$ и 3200 мкм в частотном диапазоне 0.1-50ГГц, представлены на диаграммах Смита (рис. 4). Центральное расположение $\mathrm{S}_{11}(f)$ и $\mathrm{S}_{22}(f)$ 
Восстановленные значения параметров элементов линейной эквивалентной схемы секции КЛП (частота $f=20$ ГГц)

\begin{tabular}{|c|c|c|c|c|c|c|}
\hline \multirow{2}{*}{$\begin{array}{c}\text { Длина СВЧ линии } \\
l_{W}, \text { мкм }\end{array}$} & \multicolumn{2}{|c|}{ Сопротивление } & \multicolumn{2}{|c|}{ Индуктивность } & \multirow{2}{*}{$\begin{array}{c}\text { Емкость } \\
C, \phi \Phi\end{array}$} & \multirow{2}{*}{$\begin{array}{c}\text { Проводимость } \\
G, 1 / \mathrm{OM} \\
\end{array}$} \\
\hline & $R, \mathrm{OM}$ & $D_{S}(R)$ & $L, \Gamma_{\mathrm{H}}$ & $D_{S}(L)$ & & \\
\hline 100 & 0.12 & 1.28 & $1.24 \cdot 10^{-10}$ & 1.42 & 1.41 & $1.44 \cdot 10^{-6}$ \\
\hline 200 & 0.18 & 1.24 & $7.19 \cdot 10^{-10}$ & 1.14 & 2.90 & $3.00 \cdot 10^{-6}$ \\
\hline 400 & 0.28 & 1.20 & $1.96 \cdot 10^{-9}$ & 1.03 & 5.76 & $5.81 \cdot 10^{-6}$ \\
\hline 800 & 0.69 & 1.16 & $4.23 \cdot 10^{-9}$ & 0.99 & 12.03 & $1.11 \cdot 10^{-5}$ \\
\hline 1600 & 1.65 & 1.06 & $8.12 \cdot 10^{-9}$ & 1.05 & 22.54 & $2.87 \cdot 10^{-5}$ \\
\hline 3200 & 3.43 & 1.04 & $1.68 \cdot 10^{-8}$ & 1.03 & 44.72 & $5.01 \cdot 10^{-5}$ \\
\hline
\end{tabular}

подтверждает, что разработанные КЛП обладают именно 50-омными волновыми сопротивлениями.

Для интерпретации полученных результатов рассмотрим восстановленные (экстрагированные) значения (см. таблицу) параметров элементов малосигнальной ЭС КЛП (рис. 2, b).

Из таблицы хорошо видно, что значения последовательного сопротивления скин-слоя $R$ до некоторых значений $l_{W}<\mathrm{L} \approx 800$ мкм (так называемое локальное приближение) изменяются не пропорционально изменению длины КЛП $l_{W}$. С точки зрения последовательных сопротивлений $R$ это говорит о нарушении закона Ома для участка цепи, что могло бы указывать на присутствие аномального скин-эффекта, если бы не результаты АСМ-измерений, подтверждающие наличие в исследуемых КЛП условий для реализации нормального скин-эффекта в используемом частотном диапазоне. При $l_{W} \geq \mathrm{L}$ (глобальное приближение) значения последовательных сопротивлений $R$ изменяются практически пропорционально изменению длины $l_{W}$.

Особенно сильно в локальном приближении при $l_{W}<\mathrm{L} \approx 400$ мкм размерные эффекты проявляются для параметра индуктивности $L$. В глобальном приближении $\left(l_{W} \geq \mathrm{L}\right)$ индуктивность $L$ увеличивается практически пропорционально увеличению длины КЛП $l_{W}$.

Таким образом, можно предположить, что наблюдаемые размерные эффекты могут быть связаны с фрактальной геометрией субструктуры металлизации КЛП.

Определение размерностей подобия $D_{S}$ функциональных пространств последовательных сопротивлений $\mathrm{S}(R)$ и индуктивностей $\mathrm{S}(L)$ показало наличие у них значений $D_{f}=1+D_{S}$, значительно отличающихся от топологических $D_{T}=1$, что совместно с линейными зависимостями $\ln \left(R_{0} / R_{k}\right)$ и $\ln \left(L_{0} / L_{k}\right)$ от $\ln \left(l_{W, 0} / l_{W, k}\right)$ указывает на присутствие у них фрактальных самоафинных свойств.

Согласно полученным результатам, для функционального пространства сопротивлений $S(R)$ в глобальном приближении при $l_{W} \geq \mathrm{L}$ изменение $l_{W}$ практически не приводит к значительному изменению значений $D_{f}(R) \approx(2 \pm 0.08)$ (см. таблицу). Это указывает на то, что активное сопротивление КЛП на СВЧ сигнале $(f=20$ ГГц) во многом определяется двумерной геометрией распределения неровностей рельефа поверхности скин-слоя, влияющей на латеральные процессы рас- сеяния электронов. Близкие к $D_{f}(R)$ функционального пространства $\mathrm{S}(R)$ значения $D_{f s}=2.11$ метрического пространства $\mathfrak{R}$ подтверждают это.

В локальном приближении при $l_{W}<\mathrm{L}$ уменьшение $l_{W}$ приводит к заметному увеличению значений $D_{f}(R)>2$, что указывает на возрастающую роль объема пленки, т.е. ее зеренной субструктуры, на процесс рассеяния электронов. Растущие значения $D_{f}(R)$ подтверждают это положение (см. таблицу).

Для функционального пространства индуктивностей $\mathrm{S}(L)$ уменьшение длины КЛП до $l_{W}<400$ мкм приводит к значительному увеличению значений $D_{f}(L)$ $\left(\right.$ от $D_{f}(L) \approx 2$ при $l_{W} \geq 400$ мкм до $D_{f}(L)=2.42$ для $l_{W}=100$ мкм). Это указывает на значительную роль двумерных для $l_{W} \geq \mathrm{L}$ (глобального приближения) и трехмерных для $l_{W}<\mathrm{L}$ (локального приближения) эффектов в формировании индуктивности КЛП, что не является тривиальным. Близость значения $D_{f}(L)=2.42$ функционального пространства $\mathrm{S}(L)$ со значением $D_{f g}=2.46$ метрического пространства $\mathfrak{R}$ зерен подтверждает то, что на масштабах < 400 мкм на формирование индуктивности КЛП оказывают влияние и трехмерные особенности зеренной субструктуры приповерхностной области КЛП.

\section{4. Заключение}

В работе исследовано влияние фрактальной геометрии золотой металлизации КЛП на ее аномальный скин-эффект, активное сопротивление скин-слоя $R$ и индуктивность $L$ в частотном диапазоне до 50 ГГц.

Получено, что зеренная субструктура металлизации и неоднородности рельефа поверхности КЛП являются причиной возникновения дополнительных процессов рассеяния электронов на границах зерен, которые на частотах $f>10$ ГГц переводят аномальный скин-эффект в нормальный. При этом латеральное распределение неровностей рельефа и зеренной субструктуры подчиняется законам фрактальной геометрии, что определяет нелинейное поведение $R$ и $L$ КЛП в локальном приближении при $l_{W}<\mathrm{L}$.

При этом в локальном приближении нелинейная зависимость $R$ от $l_{W}$ обеспечивается в основном фрактальной геометрией рельефа поверхности и приповерх- 
ностной области КЛП, а нелинейная зависимость индуктивности $L$ от $l_{W}$ - не только фрактальными особенностями двухмерной поверхности КЛП, но и фрактальными особенностями трехмерного расположения ее зерен. Значения пределов локальных приближений $\mathrm{L}$ для параметра активного сопротивления можно оценить как $\mathrm{L}(R) \approx 800$ мкм, а для параметра индуктивности - как $\mathrm{L}(L) \approx 400$ мкм.

\section{Финансирование работы}

Изготовление макетов и теоретическое исследование было выполнено при финансовой поддержке Министерства образования и науки РФ, базовая часть государственного задания (уникальный идентификатор FEWM2020-0046). Экспериментальные результаты получены с использованием оборудования ЦКП „Импульс“ при финансовой поддержке Министерства науки и высшего образования РФ по соглашению 075-15-2019-1644, идентификатор проекта RFMEFI62119X0029.

\section{Конфликт интересов}

Авторы заявляют, что у них нет конфликта интересов.

\section{Список литературы}

[1] В.А. Тиньков. Успехи физ. мет., 7, 117 (2006).

[2] А.В. Соколов. Оптические свойства металлов (М., Физматлит, 1961).

[3] M.I. Kaganov, P. Contreras. Zh. Eksp. Teor. Fiz., 106, 1814 (1994).

[4] А.В. Латышев, А.А. Юшканов. Журн. вычислительной математики и мат. физики, 44 (10), 1861 (2004).

[5] A.F. Mayadas, M. Shatzkes, J.F. Janak. Appl. Phys. Lett., 14 (11), 345 (1969).

[6] M.S.P. Lucas. J. Appl. Phys., 36 (5), 1632 (1965).

[7] M.A. Angadi, L.A. Udachan. Thin Sol. Films, $79(2), 149$ (1981).

[8] N.A. Torkhov. Semiconductors, 53 (1), 28 (2019).

[9] D. K. Larson. In: Physics of Thin Films, ed. by M.H. Francombe and R.W. Hoffman (N.Y., Academic, 1971) v. 6.

[10] И.В. Антонец, Л.Н. Котов, С.В. Некипелов, Е.А. Голубев. ЖТФ, 3, 24 (2004).

[11] А.Н. Колмогоров, С.В. Фомин. Элементы теории функций и функционального анализа. 4-е изд., перераб. (М., Наука, 1976).

[12] И.А. Иванишко, В.Г. Кротов. Мат. заметки, 86 (6), 829 (2009).

[13] N.A. Torkhov, L.I. Babak, A.A. Kokolov. Symmetry, 11, 1495 (2019). doi:10.3390/sym11121495

[14] N. Torkhov, L. Babak, A. Kokolov, F. Sheyerman. ITM Web of Conf., 30, 07016 (2019), CriMiCo'2019. doi.org/10.1051/itmconf /20193007016

[15] Е. Федер. Фракталь (М., Мир, 1991).

Редактор Л.В. Шаронова

\section{The influence of the microwave micro strip line surface morphology on the transmission performance}

\author{
N.A. Torkhov 1,2,3,4, A.A. Kokolov 2,5 , L.I. Babak ${ }^{2}$ \\ ${ }^{1}$ Scientific-Research Institute of Semiconductors, \\ 634034 Tomsk, Russia \\ ${ }^{2}$ Tomsk State University \\ of Control Systems and Radioelectronics, \\ 634050 Tomsk, Russia \\ 3 Tomsk State University, \\ 634050 Tomsk, Russia \\ ${ }^{4}$ Sevastopol State University, \\ 299053 Sevastopol, Russia \\ ${ }^{5}$ Zuev Institute of Atmospheric Optics, Siberian Branch \\ of Russian Academy of Sciences, \\ 634021 Tomsk, Russia
}

\begin{abstract}
The main parameters of the morphology of $50-\Omega$ $\mathrm{Au} / i$-GaAs 100 thin-film microstrip gold microwave coplanar transmission lines of length $l_{W}$ are defined, which determine the skin layer active resistance $R$ and inductance $L$. It was found that the lateral grains distribution and surface relief of the coplanar lines led to the appearance of additional processes of electron scattering both at grain boundaries and at relief inhomogeneities. When the small grain size $d x<133 \mathrm{~nm}$ at frequencies $f>10 \mathrm{GHz}$ the anomalous skin effect turns to the normal one. In this case, the nonlinear dependence of $R$ on $l_{W}$ in the local approximation was provided by the fractal geometry of the surface relief and the nearsurface region of the coplanar transmission lines, and the nonlinear dependence of the inductance $L$ on $l_{W}$ was provided not only by the fractal features of the two-dimensional coplanar transmission lines relief surface, but also by the fractal features of the threedimensional distribution of its grains.
\end{abstract}

